\title{
Consumidor de livros - variáveis determinantes do perfil do comportamento de compra no varejo on-line
}

\begin{abstract}
RESUMO
O objetivo deste estudo é identificar variáveis que mais diferenciam o perfil do comportamento de compra do consumidor de livros no varejo on-line. Para tanto, além da revisão da literatura que destaca o ambiente de mudanças rápidas no varejo, no qual a tecnologia da informação vem ganhando mais importância no relacionamento entre os consumidores e varejistas, foi realizada uma pesquisa descritiva quantitativa com cem consumidores de livros na cidade de São Paulo. O modelo de análise dos dados baseou-se na técnica de regressão logística binária. As análises indicaram que, do grupo de variáveis utilizadas na pesquisa, duas delas - quantidade de livros lidos no último ano e utilização do celular para acessar a internet - podem ser determinantes do comportamento do consumidor e orientadoras da decisão de compra de livros nas lojas on-line. Os resultados podem servir de base para novos estudos e simulações que contribuam para ampliar o conhecimento sobre os consumidores, cada vez mais conectados, e orientar os varejistas a entender e atender melhor as necessidades desses consumidores.
\end{abstract}

Palavras-chave: Varejo on-line. Comportamento de compra. Consumidor de livros.

Maria do Carmo Romeiro mromeiro@uscs.edu.br Doutora em Administração. Universidade de São Caetano do Sul.

Leandro Campi Prearo leandro.prearo@uscs.edu.br Doutor em Administração. Universidade de São Caetano do Sul.

Lidiane Campos Britto lidiane_britto@yahoo.com.br Doutoranda em Administração. Universidade de São Caetano do Sul

Mário Duarte dos Santos Machado mario.du.machado@hotmail.com Mestrando em Administração. Universidade de São Caetano do Sul

Francisco Mirialdo Chaves Trigueiro fmctrigueiro@yahoo.com.br 


\section{INTRODUÇÃO}

A internet tem se tornado parte essencial na maneira como os consumidores fazem compras, facilitadas pela convergência tecnológica presente nos aparelhos smartphones, tablets e computadores, a qual atua como integradora para que vários sistemas e aplicativos comuniquemse. Isso permite que o consumidor acesse as informações de que necessita, faça escolhas e tome decisões em tempo real, combinando as opções de compra on-line, mobile, catálogo virtual e, também, na loja física, além de várias maneiras de receber ou retirar os produtos (DELOITTE, 2011).

Com todas as facilidades geradas pelas novas tecnologias, o atual ambiente do varejo vai muito além do ambiente físico da loja. De acordo com Hofacker e Murphy (2009), os aspectos mais importantes no processo de mudança da maneira pela qual as pessoas adquirem e consomem produtos ou serviços no varejo on-line são as facilidades na busca de informações e de comparação entre as opções, informações disponíveis por meio de pesquisas nas mais diversas fontes pela Web.

Sob essa perspectiva, Huang (2008) conclui que a tomada de decisão, no processo de compra online, é influenciada por um comportamento de consumo que depende das percepções individuais do consumidor, quanto à utilidade, facilidade de uso, eficiência e segurança ao longo de todas as fases do processo.

Para o consumidor, existem algumas vantagens inquestionáveis em comprar on-line: informações ilimitadas sobre produtos e serviços, comparação instantânea de preços e custos por meio de ferramentas de pesquisa, liberdade de mudança de fornecedor ou do local onde realiza a compra e, por último, serviços ao cliente oferecidos 24 horas por dia (AHMAD; AZIZAH; RAMAYAH, 2010).

Por outro lado, os resultados da pesquisa realizada por Levin, Levin e Heath (2003) indicaram que, especificamente para as categorias de livros e CDs, os consumidores têm igual preferência por lojas on-line e lojas físicas para realizar suas pesquisas e comparar produtos, mas são fortemente inclinados a realizar suas compras nas lojas físicas.

Segundo Deloitte (2014), os varejistas dos mais diferentes segmentos de mercado devem trabalhar com a nova realidade de que os clientes de hoje têm a opção de consumir onde e quando quiserem, mesmo sem entrar na loja. Portanto, antecipar o comportamento de compra, seja no website, nas mídias sociais ou no interior da loja física e gerar experiências que cativem, é fundamental para manter o vínculo com o cliente e influenciá-lo nas suas decisões de compra, independentemente do canal de compra oferecido pelo varejista.

O processo de escolha do canal onde se dará a decisão de compra, envolve um conjunto de dimensões que levam em consideração as características individuais do consumidor, as características da compra, os critérios e atributos de avaliação do canal e a avaliação pós-consumo (BLACKWELL; MINIARD; ENGEL, 2008, p. 137).

No entanto, este artigo foca apenas em uma das dimensões, de forma a estreitar a análise sobre as características individuais do consumidor, apresentadas por Blackwell, Miniard e Engel (2008), as quais incluem o estilo de vida; as variáveis econômicas; a interação tecnológica e as experiências de compra.

Assim, compreender o comportamento de compra e a escolha dos canais pelos consumidores é relevante para os diversos produtos e serviços, sobretudo com a coexistência de canais tradicionais (ou lojas físicas) e on-line (ou lojas virtuais) de vendas. Em relação aos livros, esse é um produto que mantém características tradicionais, ao mesmo tempo em que inova com o lançamento dos e-books e utiliza-se dos canais on-line de venda. Dessa forma, estudos que permitem analisar os fatores determinantes nas decisões de compra de consumidores de livros no contexto do varejo on-line tornam-se importantes, contribuindo com análises de mudanças de perfil de compra, na detecção de novas variáveis relacionadas às compras, principalmente diante das mudanças ocasionadas pelas tecnologias móveis.

Nesse sentido, o objetivo geral do artigo é identificar as variáveis que mais diferenciam o perfil de comportamento do consumidor de livros no varejo on-line. Como objetivo específico, o artigo visa, dentre as variáveis que compõem as características individuais dos consumidores, identificar quais são as mais determinantes para a decisão de compra daquele consumidor que adquire livros no canal on-line.

Os termos loja virtual e on-line que, a partir daqui poderão ser utilizados concomitantemente, referem-se ao tipo de loja em que o cliente acessa via internet o conteúdo oferecido, realiza operações de pesquisa e de compra por meios eletrônicos. Da mesma forma, os termos loja física e loja off-line, referem-se às lojas tradicionais, com mostruários, vendedores, caixas e estoques de produtos.

A metodologia consiste em uma pesquisa descritiva e quantitativa aplicada em uma amostra não probabilística de cem clientes, abordados de forma pessoal e entrevistados na cidade de São 
Paulo, por meio de um questionário estruturado. Para o tratamento e análise dos dados, foi utilizada, principalmente, a técnica de Regressão Logística Binária por meio do software SPSS 18.

\section{O VAREJO E O VAREJO ON-LINE}

Levy e Weitz (2000, p. 27), numa definição ampliada, consideram o varejo como sendo "o conjunto de atividades de negócio que adicionam valor a produtos e serviços disponibilizados aos consumidores, para uso e consumo próprios". Considerando-se uma cadeia de valor de canais de distribuição, a loja de varejo pode ser destacada como o elo final dessa cadeia que liga fabricantes a consumidores finais, consequentemente, o elo mais próximo destes últimos que detêm o poder de compra.

Kotler e Keller (2012, p. 486) destacam que "muitos varejistas que operavam somente no canal físico, passaram também a operar no canal virtual desenvolvendo novas maneiras de comercialização". Os autores salientam um movimento importante realizado por esses varejistas, que é o de oferecer nas lojas físicas oportunidades para que o cliente vivencie novas experiências de compras e, assim, crie uma imagem de integração entre os canais físico e virtual. No que se refere às lojas de varejo, estas devem ser o local onde os consumidores podem vivenciar experiências que ativam e cativam todos os seus sentidos, tornando cada momento de compra marcante e permeável na sua mente (KOTLER; KELLER, 2012).

Por outro lado, Rosenbloom (2009, p. 365) utiliza a expressão "canais de marketing eletrônico" para destacar o que se costuma denominar de canal virtual.

O uso da Internet para tornar produtos e serviços disponíveis de tal forma que o mercadoalvo com acesso a computadores ou outras tecnologias capacitadoras, possa comprar e completar a transação de compra por meios eletrônicos interativos (ROSENBLOOM, 2009, p. 365).

Na perspectiva dos varejistas que aderem aos novos desenvolvimentos tecnológicos, Yongmei et al. (2014) observam que o desenvolvimento acelerado da tecnologia da informação e suas aplicações em mídias sociais permite que os varejistas usem o canal on-line, de forma ampla, para criar novas marcas, promover novos produtos e organizar com frequência atividades promocionais, de maneira integrada entre os canais, facilitando, assim, a fidelização do consumidor.

No Brasil, o varejo on-line apresenta crescimento, com mudanças na forma como o mercado consumidor passa a comprar produtos, conforme destacam diversas pesquisas que serão abordadas na seção seguinte.

\section{AS COMPRAS ON-LINE NO MERCADO BRASILEIRO}

Barbosa (2014) destaca que a presença de computador nas residências brasileiras apresenta uma tendência de crescimento, já verificada nos anos anteriores. Em 2013, cerca de metade dos domicílios (49\%) possuía computador e $43 \%$ deles tinham acesso à internet. Na classe $\mathrm{A}$, a porcentagem de domicílios com acesso à internet é de $98 \%$; na classe B, $80 \%$; na classe C, $39 \%$; já nas classes D e E, a porcentagem é de $8 \%$. A pesquisa destaca um aumento na proporção de usuários de internet. Entre os indivíduos de 10 a 15 anos, a porcentagem de usuários chega a $75 \%$ e, nos de 16 a 24 anos, é de $77 \%$. Na faixa de 35 a 44 anos, $47 \%$ são usuários, e na de 45 a 49 anos, a porcentagem é de $33 \%$. Por outro lado, somente $11 \%$ das pessoas com mais de 60 anos são usuárias da rede.

Barbosa (2014) também indica que, apesar do crescimento do uso de computadores nos lares brasileiros, o uso da internet pelo telefone celular está aumentando a taxas importantes. Em 2013, a estimativa é de 52,5 milhões de usuários de internet pelo telefone celular no Brasil, o que equivale a 31\% da população. Há 2 anos, esse percentual era de apenas 15\%. Nesse grupo de usuários, 30\% acessaram redes sociais do aparelho; $26 \%$ compartilharam fotos, vídeos ou textos; $25 \%$ acessaram e-mails; e $23 \%$ baixaram aplicativos. Esses dados confirmam que o uso do celular é um elemento significativo, no estudo do comportamento do consumidor que compra pela internet.

As compras eletrônicas ou e-commerce e seu crescimento são um movimento recente no Brasil, mas os números atuais demonstram a potencialidade desse canal de compras.

A Pesquisa Webshoppers, $3^{\text {a }}$ edição, realizada pela empresa E-BIT (2015), aqui utilizada como referência, mesmo com as limitações e ressalvas de ser uma amostragem do varejo on-line, aponta que o comércio eletrônico brasileiro faturou $R \$ 35$,8 bilhões em 2014, o que representa um crescimento nominal de $24 \%$ em relação a 2013 . Ao todo, foram mais de 51,5 milhões de consumidores únicos, 
sendo 10,2 milhões de novos entrantes ao longo do ano. Acrescenta-se, a essas informações, o número de 103,4 milhões de pedidos realizados, a um tíquete médio de $\mathrm{R} \$ 347,00$. As transações concretizadas por meio de aparelhos móveis corresponderam, em 2014, a 9,7\% de todas as vendas de bens de consumo pela internet.

Por outro lado, a pesquisa Webshoppers, 29a edição, da E-BIT (2014) indica que alguns fatores podem explicar o importante crescimento do comércio eletrônico no mercado brasileiro, dentre os quais, o aumento do número de domicílios com computadores, a acessibilidade por banda larga cada vez mais veloz, a melhora da segurança nas operações com meios de pagamento e o avanço no nível de serviços das operações logísticas de entrega e de troca de produtos.

Analisando especificamente a categoria livros, a pesquisa Retratos da Leitura no Brasil (FAILLA, 2012) demonstra que $50 \%$ da população brasileira têm ou tiveram acesso a livros, e quase metade desse percentual compra livros. Dos consumidores que compram livros, $35 \%$ fazem por prazer e gosto pela leitura, $32 \%$ pela cultura e $29 \%$ para entretenimento e lazer.

Nas compras on-line por categoria, a pesquisa Webshoppers $29^{a}$ edição (E-BIT, 2014) destaca que, das cinco categorias mais vendidas, a categoria livros e revistas ficou em $4^{\circ}$ lugar na preferência do consumidor, com $9 \%$, precedida das categorias moda e acessórios, com 19\%; cosméticos, perfumaria e saúde, com 18\%; eletrodomésticos, com $10 \%$ e, na frente de informática, que detém $7 \%$ do total de vendas, em número de pedidos.

Os elementos intrínsecos ao canal on-line devem continuar alimentando o crescimento do e-commerce no Brasil. Um deles é o que possibilita oferecer uma gama de produtos orientados para nichos de mercado, permitindo um número de ofertas maior, quando comparado com o canal físico. Assim, um maior número de lojas especializadas de diferentes tamanhos atuando no canal virtual permite ao consumidor escolha de opções (E-BIT, 2014).

Na perspectiva do varejo, seja on-line ou loja física, entender o comportamento de compra do consumidor é relevante, uma vez que é no varejo que acontece o contato direto do cliente final com o produto e as decisões de compra efetivam-se. Dessa forma, é com base nesse contexto teórico que será desenvolvida a próxima seção.

\section{O COMPORTAMENTO DE COMPRA DO CONSUMIDOR}

Na concepção de Chiang e Monahan (2005), diferentes clientes com distintos comportamentos de compra selecionarão o canal ou canais que melhor sirvam às suas necessidades. Além disso, conforme Goldkuhl (2007), os consumidores, quando estão comprando, podem mudar de um canal para outro em diferentes fases de uma única transação. Takahashi et al. (2011) acrescentam que a opção de comprar em diferentes canais significa, para o consumidor, mais informação, escolhas e, potencialmente, oportunidades de economia.

Blackwell, Miniard e Engel (2008, p. 137) destacam que as"escolhas realizadas pelos consumidores, são feitas a partir de percepções sobre a loja nos mais diferentes formatos, as quais constroem na sua mente um modelo formado por atributos julgados essenciais pelo indivíduo, modelo este denominado imagem de loja".

Levin, Levin e Weller (2005), em seu estudo, salientam que os consumidores tendem a buscar informações em diferentes fontes, com o objetivo de confirmarem a qualidade e garantirem o máximo de satisfação quando estão comprando diferentes tipos de produtos. Eles diferem em suas preferências por lojas físicas ou virtuais, baseados em distintos graus de importância, associados com os diversos atributos dos produtos adquiridos.

Dessa maneira, o processo de escolha envolve a combinação das características do indivíduo e do que está sendo comprado, com as características da loja. No entanto, experiências adquiridas anteriormente, mais a imagem da loja, aliadas ao processamento cognitivo automático de respostas a critérios de escolha individuais, podem acelerar o processo de tomada de decisão de compra (BLACKWELL; MINIARD; ENGEL, 2008).

O processo de escolha (Figura 1) evidencia que a escolha do canal de compra resulta da integração dos fatores, indivíduo, produto, local da compra e das avaliações pós-consumo, realimentando estas últimas a motivação para realizar novas compras. Portanto, tais "fatores orientam as análises feitas pelo consumidor para a tomada de decisão de qual tipo de varejo, qual varejista e qual canal específico ele deve comprar o produto que procura" (BLACKWELL; MINIARD; ENGEL, 2008, p. 137). 
Figura 1 - Decisão de compra: processo de escolha da loja física ou da loja on-line

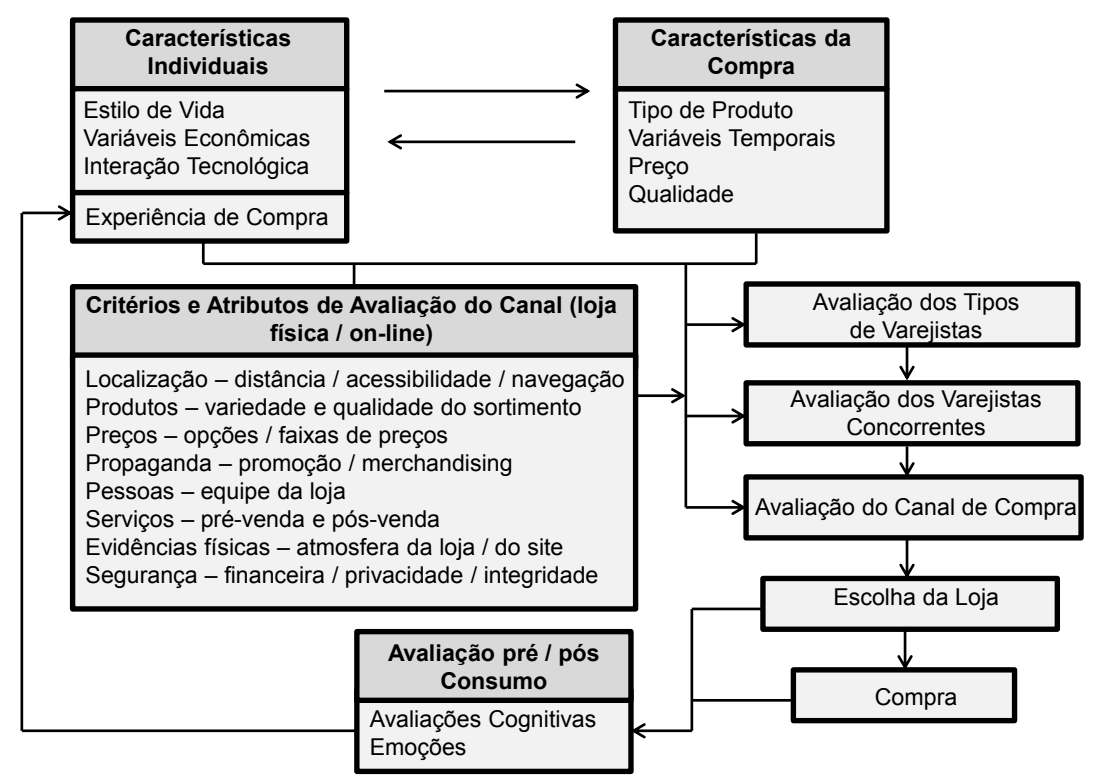

Fonte: Adaptado de Blackwell, Miniard e Engel (2008, p. 137)

Kacen, Hess e Chiang (2013) lembram que os consumidores preferem lojas que vendem produtos de alta qualidade a um preço baixo; mas eles também querem realizar suas transações rapidamente, de forma prazerosa, eficiente e segura, ou seja, visam minimizar as possíveis incertezas decorrentes dos processos que envolvem a compra.

De forma genérica, Blackwell, Miniard e Engel (2008, p. 135), após questionamento do por que razão as pessoas compram, concluíram que "os consumidores se engajam em atividades de compra, motivados por desejos de entretenimento e satisfação, pela perspectiva de fuga da rotina e até mesmo como uma forma moderna de caçada".

To, Liao e Lin (2007) reconhecem que os valores humanos relacionados ao prazer, chamados hedônicos, contribuem para ativar a motivação para o consumo; por isso, são considerados elementos motivadores de grande importância para as compras nas lojas físicas e on-line.

Bellman, Lohse e Johnson (1999) salientam que as informações referentes ao consumidor, de maior relevância para os varejistas na construção de uma previsão do comportamento de compra on-line e off-line, são aquelas que dizem respeito ao seu estilo de vida, em outras palavras, os consumidores que gastam mais on-line, são mais conectados, acessam mais a internet e interagem mais com as ferramentas de busca e avaliação disponíveis na Web.

Dessa maneira, pode-se inferir que $o$ uso do celular como instrumento do cotidiano do consumidor faz parte do seu estilo de vida e, consequentemente, do seu perfil, e pode ser uma das ferramentas para a realização das ações descritas por Bellman, Lohse e Johnson (1999).

Por outro lado, a pesquisa Retratos da Leitura no Brasil (FAILLA, 2012) confirma que as principais correlações com a leitura são: escolaridade, classe social e ambiente familiar. Isto é, quanto mais escolarizado ou mais "abastado" é o consumidor de livros, maior é a penetração da leitura e a média de livros lidos. Sendo assim, a quantidade de livros lidos pode estar relacionada às características do estilo de vida propiciadas por um poder aquisitivo maior.

Embora o comportamento de compra envolva características individuais, características do produto, critérios e atributos de avaliação do canal, dentre outros, o foco deste artigo é relacionado apenas às características individuais que compõem o perfil do comportamento de compra do consumidor, perfil este que, conforme já mencionado, pode orientar a decisão de compra no varejo on-line.

A seção seguinte apresenta os fundamentos a respeito da decisão de compra dos consumidores, de modo a identificar as características relacionadas a cada tipo de canal, on-line e off-line.

\section{A DECISÃO DE COMPRA NOS CANAIS ON-LINE E OFF-LINE}

As características individuais do consumidor on-line têm sido muito estudadas, com destaque especial para aquelas referentes ao seu perfil (MORGADO, 2003). 
Em estudo realizado por Lohse, Bellman e Johnson (2000), foi constatado que as pessoas que compram on-line têm como condição comum sofrer de escassez de tempo (time starved), mostrando que o estilo de vida seria um bom indicador para a compra on-line. Outras variáveis relacionadas ao perfil do usuário igualmente têm sido consideradas explicativas do comportamento de compra online. Ferguson e Perse (2000), em estudo realizado no mercado americano, concluíram que o nível de conhecimento no uso da internet pode ser considerado como uma das variáveis explicativas das compras pela internet.

Na perspectiva do consumidor, o canal on-line é uma importante opção de compra com características peculiares em relação ao varejo tradicional. No ambiente on-line, os consumidores são livres para fazerem compras em diferentes sites e são capazes de mudar de um para outro em apenas um clique (AHMAD; AZIZAH; RAMAYAH, 2010).

Ahmad, Azizah e Ramayah (2010) caracterizam a atividade de comprar on-line como o processo de pesquisar, investigar, procurar ou examinar um produto visando obter informações, com a finalidade de praticar a compra por intermédio da Web.

Estudos realizados por Levin, Levin e Heath (2003), sobre as preferências dos consumidores quando estão realizando suas escolhas de produtos nos canais de compra, on-line e off-line, apresentaram os resultados sumarizados na Tabela 1 a seguir, na qual os autores evidenciam que, na maioria das categorias de produtos, o canal on-line é preferido para pesquisa e comparação de atributos dos produtos, e a loja física é preferida, para algumas categorias, na fase final da compra.

Tabela 1 - Lojas on-line: percentuais de preferência de compra no canal on-line por categoria

\begin{tabular}{ccccccccc}
\hline & $\begin{array}{c}\text { Ticket } \\
\text { Aéreo }\end{array}$ & Livros & CDs & Confecção & Software & $\begin{array}{c}\text { Produtos } \\
\text { Eletrônicos }\end{array}$ & $\begin{array}{c}\text { Saúde e } \\
\text { Beleza }\end{array}$ & $\begin{array}{c}\text { Produtos } \\
\text { Esportivos }\end{array}$ \\
\hline Pesquisa & 92,5 & 50,0 & 55,0 & 22,5 & 80,0 & 50,0 & 12,5 & 30,0 \\
Comparação & 95,0 & 47,5 & 37,5 & 15,0 & 77,5 & 52,5 & 12,5 & 20,0 \\
Compra & 52,5 & 12,5 & 12,5 & 5,0 & 42,5 & 12,5 & 2,5 & 5,0 \\
\hline * & & & & & & & & \\
\end{tabular}

Fonte: Adaptado de Levin, Levin e Heath (2003)

Os resultados apresentados pelos autores demonstram os diferentes caminhos de preferência do consumidor, quanto às atividades de pesquisa, comparação e compra, das categorias de produtos. Para as categorias de confecção, artigos esportivos e produtos de saúde e beleza, os consumidores preferem, na sua grande maioria, utilizar a loja física para finalizar suas compras, quando comparados os dois canais. Para bilhetes aéreos e softwares, a preferência recai fortemente na loja on-line para a realização de pesquisas e comparações entre oportunidades de compra. $\mathrm{E}$, de forma igual, tanto online como na loja física, para a efetivação da compra. Livros, CDs e produtos eletrônicos resultaram numa resposta ligeiramente diferente. Os consumidores têm igual preferência por lojas on-line e lojas físicas para realizarem suas pesquisas e comparar produtos.

Levin, Levin e Weller (2005) concluem que a preferência para compras on-line ou off-line pode variar em razão dos produtos, dos consumidores e do estágio em que o consumidor encontra-se no que diz respeito às suas experiências de compra. As conclusões indicam que muitas das diferenças são específicas para determinadas categorias de produtos, as quais estão relacionadas ao grau de importância dado aos seus atributos, que são percebidos pelo consumidor como melhor entregues em cada um dos canais.

\section{PROCEDIMENTOS METODOLÓGICOS}

Na pesquisa, utilizou-se o método descritivo, que tem como"objetivo primordial a descrição das características de determinada população ou fenômeno ou o estabelecimento de relações entre as variáveis" (GIL, 1999, p. 44). Trata-se de uma pesquisa quantitativa, com base em uma amostra não probabilística ou de distribuição não paramétrica, com cem pessoas na cidade de São Paulo, em setembro de 2014.

Para o termo não paramétrico, Siegel (1975) atribui a denominação de distribuição livre, que tem como um de seus méritos o fato de que, ao aplicá-la, não é necessário fazer suposições a respeito da distribuição da população da qual tenham sido extraídos os dados para a análise. Como instrumento de coleta, utilizou-se um questionário estruturado, que garante certa uniformidade de uma situação de mensuração para outra (SELLTIZ et al., 1975).

Oquestionáriolevou um tempo médio de 15 minutos de aplicação para cadaum dos entrevistados, 
abordados de forma pessoal na coleta de dados. Foi dividido em três blocos estruturados: bloco 1 - perfil socioeconômico e demográfico; bloco 2 - perguntas sobre compra de livros de forma geral; e bloco 3 - perguntas acerca da internet. Para a elaboração das perguntas do questionário, os seguintes autores do referencial teórico foram utilizados: atribuição de importância de idade e escolaridade no comportamento de compra pela internet (CHIANG; MONAHAN, 2005; FAILLA, 2012); relação entre internet e compras on-line e off-line (KACEN; HESS; CHIANG, 2013; DELOITTE, 2011; AHMAD; AZIZAH; RAMAYAH, 2010; GOLDKUHL, 2007); usabilidade da internet em relação ao tempo livre e à vida cotidiana (HOFACKER; MURPHY, 2009; LEVIN; LEVIN; WELLER, 2005; BELLMAN; LOHSE; JOHNSON, 1999); relação entre leitura versus internet (FAILLA, 2012; BELLMAN; LOHSE; JOHNSON, 1999). Foi realizado um pré-teste em $5 \%$ da amostra, ou seja, foram aplicados cinco questionários. Com isso, os problemas de ordem interpretativa foram ajustados.

Para contextualizar a análise e discussão dos resultados, retomam-se os objetivos, sendo o geral: identificar variáveis que mais diferenciam o perfil de comportamento do consumidor de livros no varejo on-line; e o específico: averiguar quais variáveis relacionadas às características individuais dos consumidores são mais determinantes para a decisão de compra de livros no canal on-line.

Para analisar o perfil de comportamento do consumidor no varejo on-line, identificando quais variáveis são significativas na influência da compra, foi usada a técnica de Regressão Logística Binária, técnica esta que não depende, para o seu uso, de normalidade multivariada e de igualdade entre matrizes de variância-covariância nos grupos, e sensível a grandes amostras. Por isso, trata-se de uma técnica robusta quando esses pressupostos não são satisfeitos, tornando a sua aplicação apropriada para diversas situações (HAIR et al., 2009). Como é o caso deste artigo em que, notadamente a amostra, além de pequena, não tem normalidade multivariada.

A técnica de Regressão Logística representa dois grupos de interesse como uma variável binária com valores de 0 e 1 , e tem o objetivo de prever a probabilidade de um evento ocorrer (HAIR et al., 2009). Dessa forma, neste artigo, a variável dependente (y) da regressão logística é um valor usado para calcular a probabilidade de um consumidor comprar ou não um livro pela internet. Assim, os códigos são os seguintes: código 1 - compra de livros pela internet; código 0 - não compra de livros pela internet.

As variáveis explicativas sugeridas pelas teorias mencionadas para a composição do modelo e que integraram o questionário estão na Tabela 2.

Tabela 2 - Variáveis explicativas do modelo

\begin{tabular}{cl}
\hline NUMERAÇÃO DA VARIÁVEL $(\mathbf{X n})$ & \multicolumn{1}{c}{ VARIÁVEIS EXPLICATIVAS } \\
\hline $\mathrm{X}_{1}$ & Gênero. \\
$\mathrm{X}_{2}$ & Escolaridade. \\
$\mathrm{X}_{3}$ & Ocupação profissional. \\
$\mathrm{X}_{4}$ até $\mathrm{X}_{24}$ & O que faz no tempo livre (nav. na internet via celular, lê livros, passeia). \\
$\mathrm{X}_{25}$ & Leu livros no último mês (sim, não). \\
$\mathrm{X}_{26}$ até $\mathrm{X}_{28}$ & Quantidade de livros lidos (mês, três meses, ano). \\
$\mathrm{X}_{29}$ & Quantidade de livros comprados, em média, por ano. \\
$\mathrm{X}_{30}$ até $\mathrm{X}_{35}$ & Acessa internet de casa, trabalho, celular, lan house, casa de parentes. \\
$\mathrm{X}_{36}$ até $\mathrm{X}_{38}$ & Frequência de uso da internet (dias úteis, fim de semana, feriados). \\
$\mathrm{X}_{39}$ até $\mathrm{X}_{43}$ & Usa a internet no período da manhã, almoço, tarde, noite, madrugada. \\
$\mathrm{X}_{44}$ até $\mathrm{X}_{45}$ & Usa a internet para compras, nav. aleatoriamente, aces, redes sociais. \\
$\mathrm{X}_{55}$ até $\mathrm{X}_{65}$ & Lê pela internet sites de conteúdo aberto, livros e etc. \\
$\mathrm{X}_{66}$ & Faz compras pela internet. \\
$\mathrm{X}_{67}$ & Idade. \\
$\mathrm{X}_{68}$ & Cidade em que reside. \\
$\mathrm{X}_{69}$ & Renda familiar no último mês. \\
\hline
\end{tabular}

Fonte: Os autores (2015)

As variáveis da Tabela 2 foram submetidas a uma pré-seleção, visando identificar as menos aderentes ao modelo estatístico. Para as variáveis nominais e ordinais, foi usado o teste Qui-quadrado, e para as intervalares e razão, o teste Mann-Whitney, conforme Siegel e Castellan (2006). Referidos 
testes são mais sensíveis a pequenas amostras e, assim, adequados para análise da significância, considerando-se que são analisadas de forma bivariada.

Definiu-se como hipótese nula $\left(\mathrm{H}_{0}\right)$, nos testes de cada uma das variáveis explicativas, que a associação entre cada uma delas e a compra de livros pela internet, é nula. Dessa forma, o objetivo do teste é rejeitar $\mathrm{H} 0$. As variáveis que obtiveram $\mathrm{p}$ value $(>0,05)$ foram excluídas, por não serem significantes para a análise de regressão logística binária, caracterizada como análise multivariada, realizada num segundo momento.

A Regressão Logística Binária "não assume linearidade das relações, não assume que o erro seja normalmente distribuído, não faz exigência quanto à distribuição da normalidade multivariada, além de também relaxar a premissa de homoscedasticidade" (PREARO, 2008, p. 39). Ainda assim, eliminando as premissas anteriores, foi necessário verificar a multicolinearidade, os outliers (ausência de observações atípicas) e a relação tamanho da amostra versus as variáveis utilizadas na pesquisa.

Em relação à multicolinearidade, verificou-se que todas as variáveis significantes apresentam Variance Infraction Factor (VIF) entre um (1) e 10, o que é aceitável (HAIR et al., 2009). Outra premissa a ser considerada é a de outliers, calculada por meio da padronização da média (HAIR et al., 2009). Os resultados indicam que não existem outliers.

Após a análise das premissas iniciais, decidiu-se pelo método Enter da Regressão Logística Binária. Esse é um método no qual todas as variáveis são inseridas ao mesmo tempo em virtude da ausência de multicolienaridade problemática (HAIR et al., 2009).

Em seguida, foi necessário investigar se houve variação no -2LL. De acordo com Dias Filho e Corrar (2014), o -2LL é um indicador que busca medir a capacidade do modelo para estimar a probabilidade associada à ocorrência de determinado evento. $O$ resultado obtido no teste $-p$ value $=0,000)$ - indica que as variáveis utilizadas contribuem para a qualidade de predição do modelo. No estágio inicial, $\mathrm{o}-2 L L_{\text {null }}=117,623$ e o $-2 L L_{\text {model }}=61,186$.

De acordo com Hair et al. (2009), o pesquisador também deve construir um valor "pseudo" R2 para regressão logística semelhante ao valor R2 em análise de regressão", e que utilizam as medidas iniciais e finais do $-2 L L$ em seu cálculo. Foi encontrado o $\mathrm{R}_{\text {logit }}=48 \%$.

$\mathrm{Na}$ Regressão Logística Binária, são necessários outros testes para avaliar a qualidade do modelo, como destacam Dias e Corrar (2014), tais como o de Hosmer e Lemeshow, e o de Wald. Segundo Hair et al. (2009, p. 289), o teste de Hosmer e Lemeshow "fornece uma medida ampla de precisão preditiva que é baseada não no valor de verossimilhança, mas sim na real previsão da variável".

Por fim, estimada a equação $Z$, calcula-se a probabilidade de ocorrência (comprar ou não pela internet) por meio da fórmula $\mathrm{p}=\mathrm{e}^{\mathrm{z}} / 1+\mathrm{e}^{\mathrm{z}}$.

\section{RESULTADOS E DISCUSSÕES}

O perfil sociodemográfico dos entrevistados caracterizou-se pelas seguintes informações: $61 \%$ de pessoas do sexo feminino e $39 \%$ de pessoas do sexo masculino, com idade média de 37 anos, renda média entre $\mathrm{R} \$ 2.251,00$ e $\mathrm{R} \$ 11.250,00$. Ademais, $77 \%$ dos respondentes têm educação superior completa e $93 \%$ são residentes na cidade de São Paulo.

As variáveis significantes, resultantes da pré-seleção realizada, indicadas na Tabela 3, compõem a equação a ser estimada na Regressão Logística Binária, num total de 19. Sendo elas: $X_{2}$ - Escolaridade, $X_{11}$ - Navega na internet no tempo livre, $X_{21}$ - Desenha/pinta no tempo livre, $X_{28}$ - Quantidade de livros lidos no último ano, $X_{30}$ - Acessa internet de sua casa, $X_{31}$ - Acessa internet no trabalho, $X_{32}$ - Acessa internet no celular, $X_{36}^{30}$ - Frequência de uso da internet nos dias de semana (dias úteis), $X_{39}$ - Usa a internet no período da manhã, $\mathrm{X}_{40}$ - Usa a internet no horário de almoço, $\mathrm{X}_{44}$ - Usa a internet para fazer compras, $X_{46}$ - Usa a internet para ler (sites, revistas etc.), $X_{50}$ - Usa a internet para trabalho, $X_{51}$ - Usa a internet para atualização profissional, $X_{52}$ - Usa a internet para baixar ou ler livros, $X_{54}$ - Usa a internet para pagar contas, $X_{55}$ - Lê pela internet sites de conteúdo aberto, $X_{56}$ - Lê pela internet jornais de grande circulação, $X_{58}^{55}$ - Lê pela internet revistas. 
Tabela 3 - Variáveis significantes para o modelo

\begin{tabular}{lcc}
\hline \multicolumn{1}{c}{ VARIÁVEIS } & $Z^{*}$ & SIG $(<0,050)$ \\
\hline$X_{2}$ - Escolaridade & $-2,293$ & 0,022 \\
$X_{11}$ - Navega na internet no tempo livre & $-3,783$ & 0,000 \\
$X_{21}$ - Desenha/pinta no tempo livre & $-2,016$ & 0,044 \\
$X_{28}$ - Quantidade de livros lidos no último ano & $-2,078$ & 0,038 \\
$X_{30}$ - Acessa internet de sua casa & $-2,340$ & 0,019 \\
$X_{31}$ - Acessa internet no trabalho & $-2,487$ & 0,013 \\
$X_{32}$ - Acessa internet no celular & $-3,231$ & 0,001 \\
$X_{36}$ - Frequência de uso da internet nos dias úteis & $-4,168$ & 0,000 \\
$X_{39}$ - Usa a internet no período da manhã & $-2,020$ & 0,043 \\
$X_{40}$ - Usa a internet no horário de almoço & $-3,678$ & 0,000 \\
$X_{44}$ - Usa a internet para fazer compras & $-3,729$ & 0,000 \\
$X_{46}$ - Usa a internet para ler (sites, revistas etc) & $-2,972$ & 0,003 \\
$X_{50}$ - Usa a internet para trabalho & $-2,406$ & 0,016 \\
$X_{51}$ - Usa a internet para atualização profissional & $-2,117$ & 0,034 \\
$X_{52}$ - Usa a internet para baixar ou ler livros & $-2,135$ & 0,033 \\
$X_{54}$ - Usa a internet para pagar contas & $-2,531$ & 0,011 \\
$X_{55}$ - Lê pela internet sites de conteúdo aberto & $-1,965$ & 0,049 \\
$X_{56}$ - Lê pela internet jornais de grande circulação & $-2,514$ & 0,012 \\
$X_{58}$ Lê pela internet revistas & $-2,429$ & 0,015 \\
\hline
\end{tabular}

* Teste Mann-Whitney

Fonte: Os autores (2015)

Assim, a equação a ser estimada é:

$Z=$ Constante $+\beta_{1} X_{2}+\beta_{2} X_{11}+\beta_{3} X_{21}+\beta_{4} X_{28}+\beta_{5} X_{30}+\beta_{6} X_{31}+\beta_{7} X_{32}+\beta_{8} X_{36}+\beta_{9} X_{39}+\beta_{10} X_{40}+$ $\beta_{11} X_{44}+\beta_{12} X_{46}+\beta_{13} X_{50}^{1}+\beta_{14} X_{51}+\beta_{15} X_{52}^{21}+\beta_{15}^{4} X_{54}^{28}+\beta_{17}^{5} X_{55}^{30}+\beta_{18}^{6} X_{56}+\beta_{19}^{32} X_{58}$

As variăveis relacionadas à internet são as que aparecem em maior proporção no modelo como significantes, o que corrobora com a premissa de que a internet torna os produtos e serviços mais disponíveis para compra (KACEN; HESS; CHIANG, 2013; DELOITTE, 2011; AHMAD; AZIZAH; RAMAYAH, 2010; ROSENBOOM, 2009; GOLDKUHL, 2007). Além disso, com base na análise feita, pôde-se perceber também que as variáveis local de acesso, frequência da utilização da internet no tempo livre e no cotidiano tendem a afetar o comportamento de compra do consumidor (HOFACKER; MURPHY, 2009; BELLMAN; LOHSE; JOHNSON, 1999).

Com base nos resultados da Regressão Logística Binária, atendidas as premissas descritas na metodologia, identificou-se que apenas duas variáveis explicam a equação final, conforme a Tabela 4. As variáveis são: $X_{28}$ - Quantidade de livros lidos no último ano (estatística de Wald = 3,969 e Sig. 0,046 ) e $X_{32}$ - Acesso da internet pelo celular (estatística de Wald = 4,604 e Sig. 0,032).

Tabela 4 - Variáveis da Equação de Regressão Logística

\begin{tabular}{|c|c|c|c|c|}
\hline VARIÁVEL & $\beta$ & $\begin{array}{c}\text { Erro } \\
\text { Padrão }\end{array}$ & $\begin{array}{l}\text { Teste } \\
\text { Wald }\end{array}$ & Sig.* \\
\hline $\mathrm{X}_{2}-$ Escolaridade & $-3,342$ & 3,624 & 0,850 & 0,356 \\
\hline$X_{11}$ - Navega na internet no tempo livre & 1,123 & 0,800 & 1,971 & 0,160 \\
\hline$X_{21}$ - Desenha/pinta no tempo livre & $-0,706$ & 0,467 & 2,285 & 0,131 \\
\hline$X_{28}$ - Quantidade de livros lidos no último ano & 0,209 & 0,105 & 3,969 & 0,046 \\
\hline$X_{30}$ - Acessa internet de sua casa & $-1,355$ & 0,880 & 2,372 & 0,124 \\
\hline$X_{31}-$ Acessa internet no trabalho & $-0,197$ & 0,448 & 0,193 & 0,661 \\
\hline$X_{32}$ - Acessa internet no celular & 0,971 & 0,452 & 4,604 & 0,032 \\
\hline $\mathrm{X}_{36}$ - Frequência de uso da internet nos dias úteis & 19,238 & 14403,90 & 0,000 & 0,999 \\
\hline $\mathrm{X}_{39}$ - Usa a internet no período da manhã & $-0,091$ & 0,141 & 0,423 & 0,516 \\
\hline $\mathrm{X}_{40}$ - Usa a internet no horário de almoço & 0,203 & 0,119 & 2,906 & 0,088 \\
\hline $\mathrm{X}_{44}$ - Usa a internet para fazer compras & 0,069 & 0,136 & 0,256 & 0,613 \\
\hline $\mathrm{X}_{46}$ - Usa a internet para ler (sites, revistas etc.) & 0,158 & 0,188 & 0,702 & 0,402 \\
\hline$X_{50}$ - Usa a internet para trabalho & 0,251 & 0,246 & 1,043 & 0,307 \\
\hline$X_{51}$ - Usa a internet para atualização profissional & $-0,001$ & 0,192 & 0,000 & 0,994 \\
\hline$X_{52}$ - Usa a internet para baixar ou ler livros & $-0,129$ & 0,136 & 0,892 & 0,345 \\
\hline$X_{54}$ - Usa a internet para pagar contas & $-0,025$ & 0,116 & 0,048 & 0,827 \\
\hline$X_{55}$ - Lê pela internet sites de conteúdo aberto & 0,035 & 0,178 & 0,039 & 0,844 \\
\hline$X_{56}$ - Lê pela internet jornais de grande circulação & $-0,091$ & 0,170 & 0,285 & 0,594 \\
\hline$X_{58}$ - Lê pela internet revistas & 0,110 & 0,134 & 0,673 & 0,412 \\
\hline Constante & $-80,318$ & 57615,62 & 0,000 & 0,999 \\
\hline
\end{tabular}

Fonte: Os autores (2015) 
A equação final do modelo é: $Z=-80,318+0,209 X_{28}+0,971 X_{32}$

Os resultados para o teste de Hosmer e Lemeshow indicaram que os valores preditos não são significativamente diferentes dos observados, caracterizando a qualidade do modelo. Nesse sentido, o modelo pode ser utilizado para estimar a probabilidade da compra ou não compra de livros pela internet em razão das variáveis independentes.

Desse modo, o acerto de quem não compra pela internet foi de $69 \%$. O acerto de quem compra foi de $100 \%$. Assim, o índice de acerto geral é de $90,6 \%$. Sendo assim, pode-se concluir que, com base nesses resultados, é estatisticamente viável incluir as variáveis independentes no modelo, uma vez que tendem a explicar o status assumido para cada consumidor (compra ou não compra livros pela internet).

Embora o índice de acerto encontrado apresente um valor alto, ressalta-se que em virtude do tamanho da amostra e da não generalização dos resultados, por causa da amostra não ser probabilística, há probabilidade de que existam vieses. Entretanto, o modelo equacional direciona para as variáveis mais determinantes na decisão de compra de livros no varejo on-line, indicando que estudos futuros com novos planos amostrais possam relacionar outras variáveis significativas, préselecionadas nesse estudo, para comporem a equação final do modelo.

Bellman, Lohse e Johnson (1999) ressaltam que aqueles consumidores com um perfil de compra que tendem mais ao on-line, são mais conectados, acessam mais a internet e interagem mais com as ferramentas de busca e avaliação disponíveis na Web. Na mesma linha, o resultado das análises demonstrou que a variável $X_{32}$ - acessa internet pelo celular -, significante no modelo, é um influenciador do comportamento de compra do consumidor, conforme indicam Blackwell, Miniard e Engel (2008). Dessa forma, a convergência tecnológica presente em aparelhos smartphones, tablets e computadores, de acordo com Delloitte (2011), permite que o consumidor acesse as informações de que necessita, faça escolhas e tome decisões em tempo real, combinando as opções de compra on-line, mobile, catálogo virtual e também na loja física, além de várias maneiras de receber ou retirar os produtos.

Os achados do estudo tendem a corroborar com pesquisa realizada por Ferguson e Perse (2000), na qual concluíram que o nível de conhecimento no uso da internet pode ser considerado como uma das variáveis explicativas das compras pela rede. Nesse sentido, o acesso constante à internet, sobretudo pelo celular ou outras plataformas móveis, é um indicador do conhecimento do consumidor sobre como comprar pela rede, sendo uma variável que contribui na decisão de compra no varejo on-line.

Blackwell, Miniard e Engel (2008), após questionamentos a respeito das razões que levam as pessoas a comprarem, concluíram que os consumidores engajam-se em atividades de compra, motivados por desejos de entretenimento e satisfação. Das características individuais objeto do estudo e destacadas pelos autores, na Figura 1, estilo de vida e interação tecnológica guardam uma relação próxima com entretenimento e satisfação.

A leitura de livros é uma maneira de entretenimento e compõem o perfil de comportamento ligado ao estilo de vida do consumidor (FAILLA, 2012).

Por outro lado, na medida em que o consumidor tem as facilidades da interação tecnológica oferecida pelas plataformas móveis, dentre elas o celular, e confia nas diversas fases do processo de compra, segundo Huang (2008), ele tem uma predisposição maior de realizar compras e, assim, satisfazer seus desejos. Portanto, como resultado obtido pelo modelo as variáveis mais significativas, quantidade de livros lidos e acesso à internet pelo celular, convergem para as características individuais, destacadas por Blackwell, Miniard e Engel (2008), estilo de vida e interação tecnológica, que compõem o perfil do comportamento de compra do consumidor, que no estudo foca o consumidor que compra livros on-line.

Assim, na compra de livros pela internet, as tecnologias móveis permitem que esse processo ocorra independentemente de hora e lugar, facilitando a tomada de decisão de compra.

\section{CONCLUSÕES}

Esta pesquisa abordou o tema da escolha do varejo on-line e/ou físico como possíveis canais de compra de livros. Para tanto, o objetivo proposto foi o identificar variáveis que mais diferenciam o perfil de comportamento do consumidor de livros no varejo on-line.

Identificou-se no início da análise que uma amostra maior poderia tornar o modelo com uma qualidade melhor para predizer valores e calcular a probabilidade do cliente comprar ou não pela internet, devido ao número inicial de variáveis significativas, separadas por testes de significância como o Qui-Quadrado e o Mann-Whitney. 
As principais análises conclusivas foram que apenas duas variáveis $\left(X_{28}\right.$ e $\left.X_{32}\right)$ têm poder expressivo de explicação do modelo, levando-se em conta uma significância de $5 \%$. As variáveis são: quantidade de livros lidos no último ano e acesso à internet pelo celular. O modelo tem assim razoável poder de explicação, mas pode ser aprimorado com o aumento da amostra.

Como se trata de um modelo que aufere questões relativas ao comportamento do consumidor, estimando a compra ou não compra de livros pela internet e levando-se em conta que apenas duas variáveis são significativas para o modelo, o resultado mostra que, de acordo com o modelo da equação final, o acerto de quem não compra pela internet foi de $69 \%$. O acerto de quem compra foi de $100 \%$. Assim, o índice de acerto geral é de $90,6 \%$.

As discussões relativas ao comportamento de compra do consumidor há muito tem sido alvo das discussões acadêmicas e mercadológicas, principalmente por conta do crescente número de canais de venda no varejo. Em tempos de dúvidas em relação à mudança de suporte dos meios impressos para os meios virtuais, contatou-se, pela pesquisa Retratos de Leitura no Brasil (FAILLA, 2012), que o número de leitores no Brasil caiu de 95,6 milhões para 88,2 milhões. Dessa forma, entender as transformações do perfil do consumidor de livros, por meio da escolha de um canal de compra, pode contribuir para que o mercado possa preparar respostas mais adequadas aos cenários futuros.

\title{
BOOK CONSUMER - DETERMINANT VARIABLES OF PURCHASE BEHAVIOR PROFILE IN ONLINE RETAIL
}

\begin{abstract}
The aim of this study is to identify determinant variables of behavior profile in the purchase of books in online retail. Therefore, in addition to literature review that highlights the environment of rapid change in retail, where information technology is gaining more importance in the relationship between consumers and retailers, a quantitative descriptive survey of 100 book consumers was carried out in the city of Sao Paulo. The data analysis model was based on binary logistic regression. Analyses indicated that among the variables used in the research group, two of them - the quantity of books read in the last year and the use of mobile phone to access the internet - may be influencing consumer behavior and guiding book purchase decision in online stores. The results can serve as a basis for further studies and simulations that contribute to increase the knowledge about consumers, who are more and more connected, and guide retailers to understand them and meet their needs.
\end{abstract}

Keywords: Online retail. Purchase behavior. Book consumer. 


\section{REFERÊNCIAS}

AHMAD, Norzieiriani; AZIZAH, Omar; RAMAYAH, T. Consumer lifestyles and online shopping continuance intention. Business Strategy Series, Bradford, v. 11, n. 4, p. 227-243, 2010.

BARBOSA, Alexandre F. (Coord.). TIC Domicílios e empresas 2013. [2014]. Disponível em: <http:// www.cetic.br>. Acesso em: 2 mar. 2015.

BELLMAN, Steven; LOHSE, Gerald.L.; JOHNSON, Eric J. Predictors of online buying behavior. Communication of the ACM, New York, v. 42, n. 12, p. 32-38, 1999.

BLACKWELL, Roger D.; MINIARD, Paul W.; ENGEL, James F. Comportamento do consumidor. 9. ed. São Paulo: Cengage Learning, 2008.

CHIANG, Wey-yu Kevin; MONAHAN, George E. Managing inventories in a two enchant dual channel supply chain. European Journal of Operational Research, Amsterdan, v. 162, n. 2, p. 325-341, Apr. 2005.

DELOITTE. Serving the connected consumer: The Deloitte Consumer Review. [2011]. Disponível em :<http://www.deloitte.com>. Acesso em: 5 out. 2014.

DELOITTE. The new digital divide: Retailers, shoppers, and the digital influence factor. [2014]. Disponível em: <http://www.deloitte.com/us/Digitallnfluence>. Acesso em: 5 out. 2014.

DIAS FILHO, Jose Maria; CORRAR, Luiz João. Regressão Logística. In: CORRAR, L.J.; PAULO, E.; DIAS FILHO, J.M. (Coord.). Análise multivariada para os cursos de administração, ciências contábeis e economia. São Paulo: Atlas, 2014. cap. 5.

E-BIT. Pesquisa Webshoppers 29a edição. [2014]. Disponível em: <www.webshoppers.com.br>. Acesso em: 6 jun. 2014.

E-BIT. Pesquisa Webshoppers $31^{\text {a }}$ edição. [2015]. Disponível em: <www.webshoppers.com.br>. Acesso em: 24 fev. 2015.

FAILLA, Zoara (Org.). Retratos da leitura no Brasil. 3. ed. São Paulo: Instituto Pró Livro, 2012. Disponível em: <http://www.prolivro.org.br/ipl/publier4.0/dados/anexos/2834_10.pdf. >. Acesso em: 17 out. 2014.

FERGUSON, Douglas A.; PERSE, Elizabeth M. The World Wide Web as a Functional Alternative to Television. Journal of Broadcasting \& Electronic Media, Washington, v. 44, n. 2, p. 155-174, 2000.

GIL, Antonio Carlos. Métodos e técnicas de pesquisa social. 5. ed. São Paulo: Atlas, 1999.

GOLDKUHL, Lena. Multiple marketing channel conflict with a focus on the internet: A Dual Perspective. 2007. Ph.D. Dissertation (Ph.D.) - Lulea University of Technology, Sweden.

HAIR JR, Joseph F. et al. Análise multivariada de dados. 6. ed. São Paulo: Bookman, 2009.

HOFACKER, Charles F.; MURPHY, Jamie. Consumer web page search, clicking behavior and reaction time. Direct Marketing - An International Journal, Texas, v. 3, n. 2, p. 88-96, 2009.

HUANG, Echo. Use and gratification in e-consumers. Internet Research, Hackensack, NJ, USA, v. 18, n. 4, p. 405-426, 2008.

KACEN, Jacqueline J.; HESS. James D.; CHIANG, Wei-yu Kevin. Bricks or Clicks? Consumer attitudes toward traditional stores e online stores. Global Economics and Management Review, [S.I.], v.18, n. 1, p.12-21, Jan./Apr. 2013.

KOTLER, Philip; KELLER, Kevin Lane. Administração de marketing. 14. ed. São Paulo: Pearson Education do Brasil, 2012.

LEVIN, Aron M.; LEVIN, Irwin P.; HEATH, C. Edward. Product category dependent consumer preferences for online and offline shopping features and their influence on multichannel retail alliances. Journal of Electronic Commerce Research, California, USA, v. 4, n. 3, 2003, p. 85-93. 
LEVIN, Aron M.; LEVIN, Irwin P.; WELLER, Joshua. A. A multi-attribute analysis of preferences for online and offline shopping: differences across product, consumers, and shopping stages. Electronic Commerce Research, New York, v. 6, n. 4, p. 281-290, 2005.

LEVY, Michael; WEITZ, Barton. Administração de varejo. São Paulo: Atlas, 2000.

LOHSE, Gerald L.; BELLMAN, Steven; JOHNSON, Eric J. Consumer Buying Behavior on the Internet: Findings from Panel Data. Journal of Interactive Marketing, [S.I.], v. 14, n. 1, p. 15-29, 2000.

MORGADO, Maurício Gerbaudo. Comportamento do consumidor online: perfil, uso da Internet e atitudes. 2003. Tese (Doutorado) - Fundação Getúlio Vargas, São Paulo.

PREARO, Leandro Campi. $\mathbf{O}$ uso de técnicas estatísticas multivariadas em dissertações e teses sobre o comportamento do consumidor: um estudo exploratório. 2008. Dissertação (Mestrado) Faculdade de Economia, Administração e Contabilidade, Universidade de São Paulo, São Paulo.

ROSENBLOOM, Bert. Canais de marketing: uma visão gerencial. São Paulo: Atlas, 2009.

SELLTIZ, Claire et al. Métodos de pesquisa nas relações sociais. São Paulo: EPU, 1975.

SIEGEL, Sidney. Estatística não-paramétrica para as ciências do comportamento. São Paulo: McGraw-Hill, 1974.

SIEGEL, Sidney; CASTELLAN Jr., N. John. Estatística Não-Paramétrica para Ciências do Comportamento. 2. ed. Porto Alegre: Artmed, 2006.

TAKAHASHI, K. et al. Inventory control in a two-echelon dual-channel supply chain with setup of production and delivery. International Journal Production Economics, Amsterdan, n. 133, p. 403$415,2011$.

TO, Pui-Lai; LIAO, Chechen; LIN, Tzu-Hua. Shopping motivations on internet: a study based on utilitarian and hedonic value. Technovation, Essex, England, n. 27, p. 774-787, 2007.

YONGMEI Liu et al. Pricing decision under dual-channel structure considering fairness and freeriding behavior. Hindawi Publishing Corporation Discrete Dynamics in Nature and Society, New York, v. 2014, p. 1-10, May 2014. 